\title{
Hidden Problems in Precise Calibration on Microstrip
}

\author{
Jiri Raboch, Karel Hoffmann, Zbynek Skvor and Premysl Hudec \\ Czech Technical University in Prague, Faculty of Electrical Engineering, \\ Technicka 2, 16627 Prague 6, Czech Republic \\ E-mail: hoffmann@fel.cvut.cz,rabocj1@ fel.cvut.cz, skvor@fel.cvut.cz
}

\begin{abstract}
A study of calibration problems on microstrip with respect to interaction of electromagnetic fields around SMA to microstrip launcher and microstrip short was carried out in frequency band up to $26 \mathrm{GHz}$. Omni Spectra SMA to microstrip launcher, $50 \Omega$ microstrip line on Arlon CuClad $2230.508 \mathrm{~mm}$ thick substrate and microstrip shorts with reflective wall above the upper side of substrate as a calibration elements were used for simulations in CST Microwave Studio. Strong influence of dimensions of shorts and distance between the shorts and the launcher on simulated calibration data was observed. Reasonable dimensions of the short and the distance between the short and the launcher making possible correct precise calibration were determined.
\end{abstract}

\section{INTRODUCTION}

Many kinds of correction methods for s-parameter vector measurements have been developed since Hackborn and Rehnmark suggested a calibration process for vector network analyzer (VNA) systems, [1], [2]. In all calibration methods values of elements of various error models are determined on the basis of measurement of calibration data obtained by measurement of calibers like Open, Short, Load, Thru, Line, etc. It is always supposed and necessarily demanded so that values of elements of an error model were constant during calibration and subsequent measurement. Surprisingly great systematic errors can be removed in such cases. The demand of constant values of elements error model also means that they are independent on the caliber connected to VNA. It is simple to satisfy in non radiating structures like a coaxial line. However, the situation is more complicated in case of measurement on an open structure like a microstrip line. A launcher connecting the microstrip line with the coaxial line of a port of the VNA more or less radiates and some higher order modes occur in the vicinity of the launcher. If a caliber is placed too close to this area the electromagnetic field around the launcher can interact with that of the caliber. Such an interaction may differ during calibration and measurement and thus may result in strong changes of calibration data obtained by measurement of the caliber, and dramatic increase of measurement uncertainty.

The purpose of this paper is to clear up this issue and to give some practical recommendation to minimize these problems.

The analysis was done for Omni Spectra SMA to microstrip launcher, see Fig. 1, connected to a $50 \Omega$ microstrip line realized on Arlon CuClad 2230.508 mm thick substrate with $\varepsilon_{\mathrm{r}}=2.33$. CST Microwave Studio was used for simulations.

First of all simulations of reflection coefficient at the coaxial part of the launcher for different dimensions of the microstrip Short and different distances between the Short and the launcher were carried out. Then dimensions of a microstrip Short providing satisfactory electrical parameters were determined. Finally with this Short placed in different distances from the launcher the reflection coefficients at the coaxial part of the launcher were simulated.

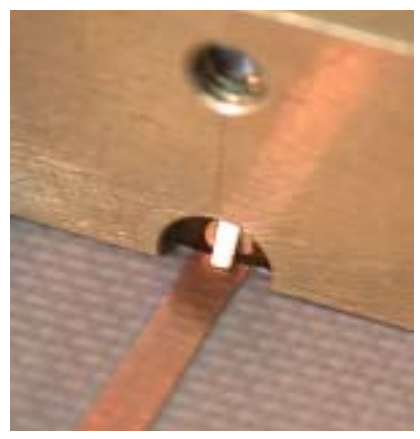

Fig. 1. SMA to microstrip launcher.

\section{FIRST SIMULATIONS}

The first simulation was made with the microstrip Short placed at the edge of the microstrip at the distance of $25 \mathrm{~mm}$ from the launcher, see Fig. 2. The height of the metal sheet above the upper side of the substrate was $5 \mathrm{~mm}$ and the width was $20 \mathrm{~mm}$.

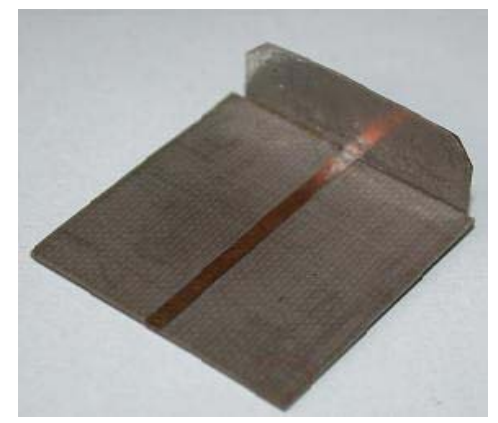

Fig. 2. Microstrip Short. 
Fig. 3 displays magnitude of electric field in the structure at frequency $22.8 \mathrm{GHz}$. The structure clearly radiates especially when the maximum of electric intensity standing wave on the microstrip occurs on the launcher. Simulations have revealed that up to $30 \%$ of energy fed into SMA connector may leak out of the structure.
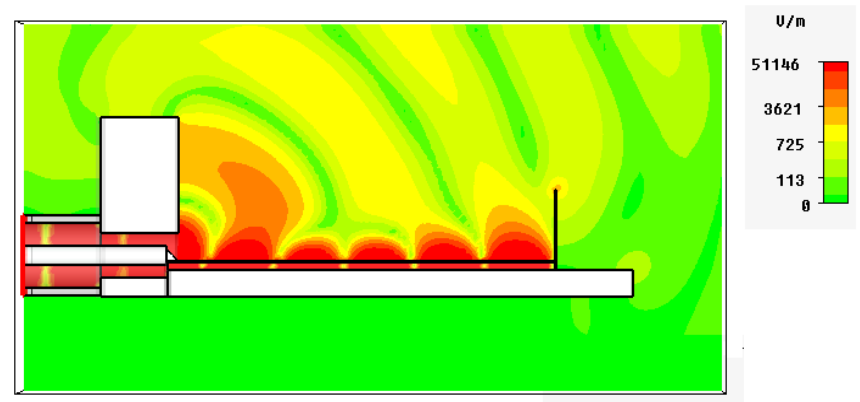

Fig. 3. Distribution of magnitude of electrical field in the analyzed structure at frequency $22.8 \mathrm{GHz}$.

Further simulations were made for different heights of the microstrip Shorts. The width of the metal sheet was $23 \mathrm{~mm}$. The highs of the Shorts above the upper side of the substrate were $0 \mathrm{~mm}, 2.5 \mathrm{~mm} .5 \mathrm{~mm}$ and $10 \mathrm{~mm}$. The results of simulations are displayed in Fig. 4 to Fig. 6

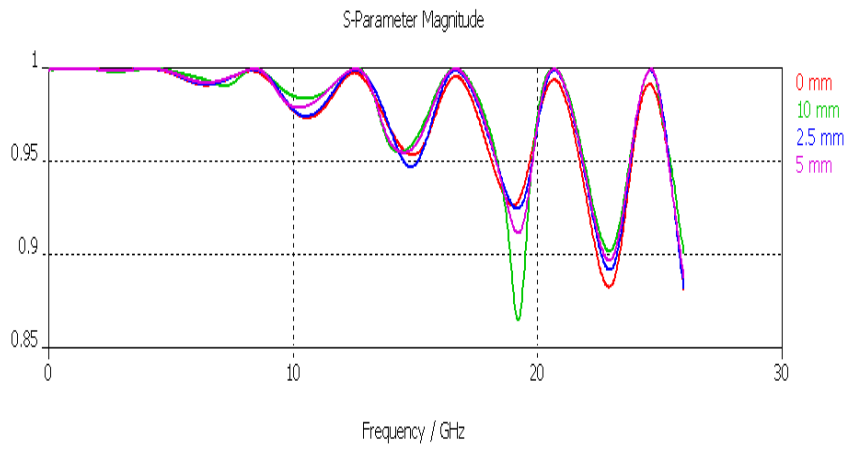

Fig. 4. Reflection coefficient at the input of the launcher of microstrip Shorts with different metal sheet height placed at the distance $25 \mathrm{~mm}$ from the launcher.

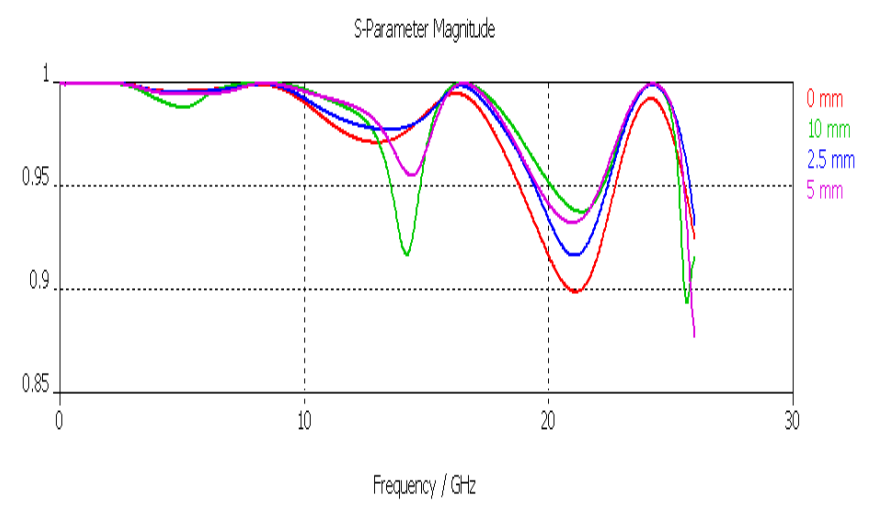

Fig. 5. Reflection coefficient at the input of the launcher of microstrip Shorts with different metal sheet height placed at the distance $12.5 \mathrm{~mm}$ from the launcher.

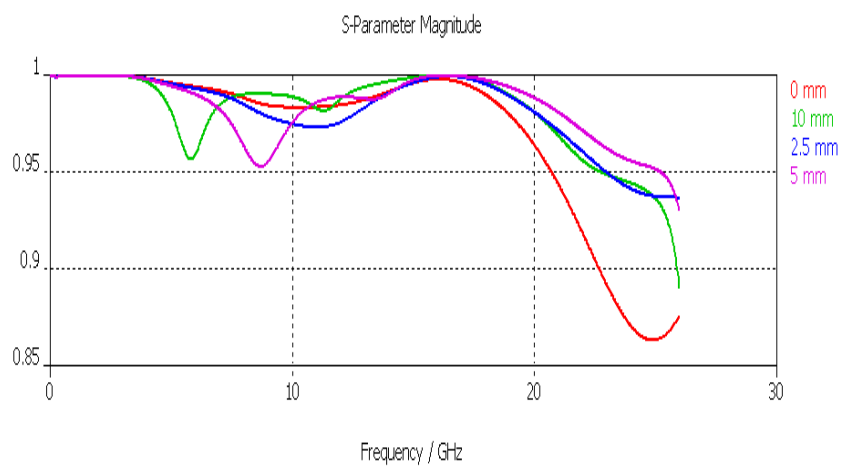

Fig. 6. Reflection coefficient at the input of the launcher of microstrip Shorts with different metal sheet height placed at the distance $6.25 \mathrm{~mm}$ from the launcher.

It can be seen that Shorts with different height create different reflection coefficients. The phenomenon is more significant for Shorts close to the launcher. It is obvious that some interactions between near field and also radiated field of the launcher and the Short occur. However the differences in reflection coefficients can be caused both the interaction of the field of the launcher and different (may be insufficient) dimensions of the Shorts. Therefore it was decided to determine dimensions of the metal sheet of the Short to form sufficiently good electrical properties of the Short.

\section{MICROSTRIP SHORT}

Proper dimensions of the Short were determined by simulation. Shorts with different height were placed at the edge of the substrate. Reflection coefficients calculated at the distance $25 \mathrm{~mm}$ from the Shorts at microstrip line were calculated and transformed to the reference plane of the shorts using CST Microwave Studio, see Fig. 7. It can be seen the shorts with height of metal plane equal or higher than $5 \mathrm{~mm}$ have practically acceptable parameters. Therefore the Short with the height of the metal sheet $5 \mathrm{~mm}$ was chosen for further simulations.

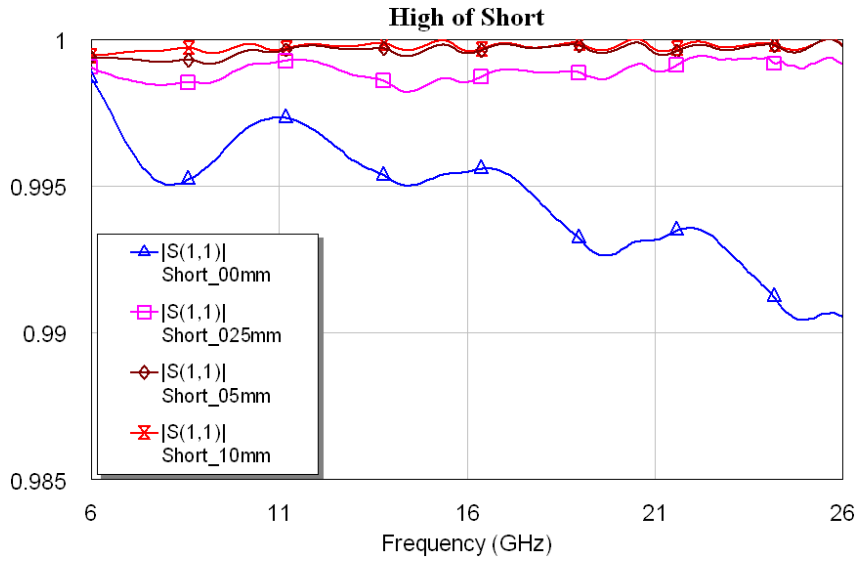

Fig. 7. Microstrip Shorts reflection coefficients magnitudes with different heights of metal sheet. 


\section{POSITIONING OF SHORT}

A short with proper dimensions does its job in calibration well as far as its electromagnetic field does not interact with other parts of calibration setup. Having obtained dimensions of the Short, minimum distance of the short from the launcher remains to be determined.

The whole structure consisting from the launcher connected to the Short through a microstrip of certain length can be considered as one object and analyzed by the electromagnetic field simulator. However, it can be also considered as a cascade connection of two blocks - the launcher with the microstrip line as the first block and the Short as the second block. The later approach neglects electromagnetic coupling.

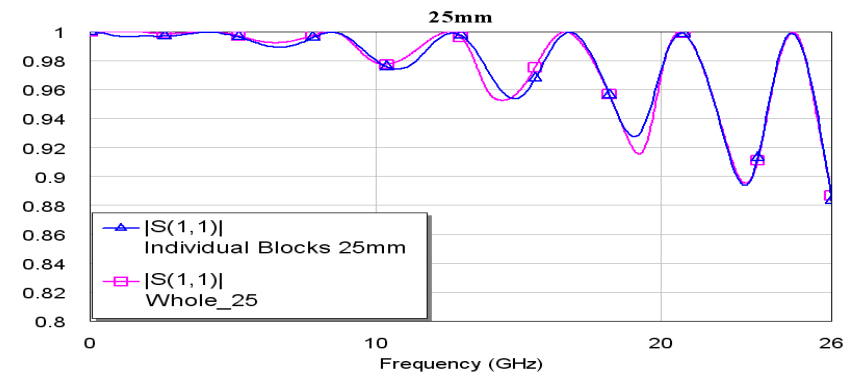

Fig. 8. Reflection coefficient of the cascade of two blocks compared to the reflection coefficient of the whole structure. The length of the microstrip is $25 \mathrm{~mm}$.

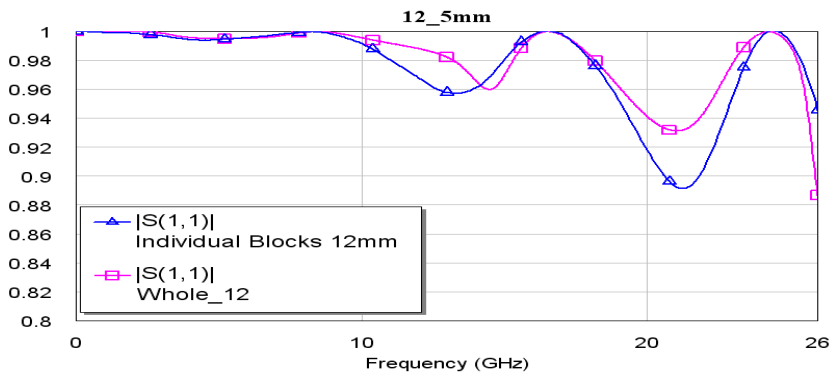

Fig. 9. Reflection coefficients of the cascade of two blocks compared to the reflection coefficient of the whole structure. The length of the microstrip is $12.5 \mathrm{~mm}$.

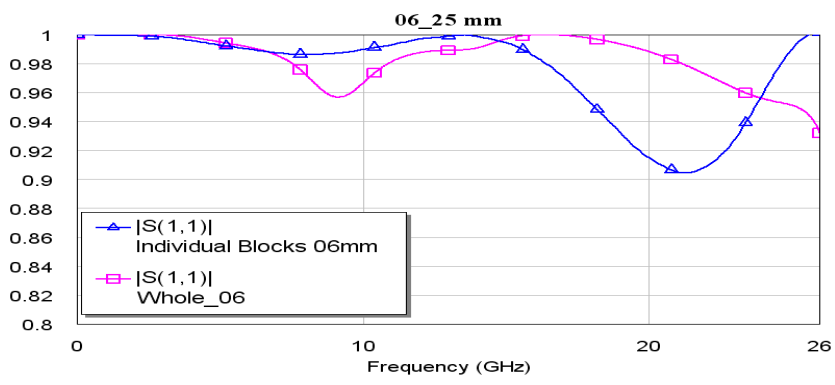

Fig. 10. Reflection coefficients of the cascade of two blocks compared to the reflection coefficient of the whole structure. The length of the microstrip is $6.25 \mathrm{~mm}$.
Both blocks can be simulated to obtain their s-parameters and then calculate s-parameter (reflection coefficient) of the cascade using some circuit simulator, AWR Microwave Office in our case. Simulated reflection coefficients at the input of the launcher should be identical for the whole structure and for both blocks in cascade in case that the Short does not influence the electromagnetic field distribution near the launcher. Comparing results of both simulations minimum distance between the launcher and the Short can be determined.

The results of simulations show Fig. 8 to Fig. 10. It can be seen that distances 12.5 and $6.25 \mathrm{~mm}$ are not reasonable. The difference caused by direct coupling in the case of $25 \mathrm{~mm}$ spacing is negligible when compared to other errors, and therefore the $25 \mathrm{~mm}$ spacing proved to be enough.

\section{CONCLUSION}

Microwave measurements in open structures require good knowledge of electromagnetic effects. It has been observed that the structure consisting of coaxial to microstrip launcher, a piece of microstrip line and a microstrip short may exhibit strong radiation and mutual couplings. These couplings between launcher and calibers that can not be easily implemented into systematic error correction schemes should be minimized during measurement setup design. Fullwave electromagnetic simulation proved to be an efficient way to provide for that.

Experimental study has been carried out for OmniSpectra SMA microstrip launcher and a $50 \mathrm{ohm}$ microstrip line on Arlon CuClad233 $0.508 \mathrm{~mm}$ thick substrate. $5 \mathrm{~mm}$ height of the metal plane and a minimum spacing of $25 \mathrm{~mm}$ between the launcher and the Short have been found as a reasonable recommendation for practical measurements. It is suggested to use a similar algorithm for other calibers.

\section{ACKNOWLEDGEMENT}

This work has been conducted at the Department of Electromagnetic Field of the Czech Technical University in Prague and supported by the research program MSMT6840770015 "Research of Methods and Systems for Measurement of Physical Quantities and Measured Data Processing" of CTU in Prague sponsored by the Ministry of Education, Youth and Sports of the Czech Republic and by the Czech Science Foundation under Grant 102/08/H027 "Advanced Methods, Structures and Components of Electronic Wireless Communication" (presentation).

\section{REFERENCES}

[1] R. A. Hackborn, "An automatic network analyzer system," Microwave J.. pp 45-52, May 1968.

[2] S. Rehnmark, "On the Calibration Process of Automatic Network Analyzer Systems," IEEE Trans. on MTT, vol. MTT22, pp. 457-458, April 1974. 